\title{
Comparative Study of Heart Rate Extraction Methods for a Novel Intelligent Mattress
}

\author{
Samuel Otis*, Neila Mezghani*† and Bessam Abdulrazak ${ }^{\ddagger}$ \\ *Laboratoire de recherche en imagerie et en orthopédie, Centre de recherche du CHUM \\ École de technologie supérieure, Montreal, Canada \\ Email: samuel.otis.1@ens.etsmtl.ca \\ ${ }^{\dagger}$ Centre de recherche LICEF, Université TÉLUQ, Montreal, Canada \\ Email: neila.mezghani@teluq.ca \\ ${ }^{\ddagger}$ Dept. of Computer Science, Sherbrooke University, Sherbrooke, Canada \\ Email: Bessam.Abdulrazak@usherbrooke.ca
}

\begin{abstract}
Unobtrusive monitoring of the heart rate (HR) is essential for improving medical intervention. A new generation of mattress-based fiber optic sensor (FOS) is emerging for HR monitoring. The use of this FOS mattress for medical diagnosis requires appropriate advanced signal processing algorithms. In our study, we aim to weigh the performances of a novel and cheaper microbend FOS mattress by applying ballistocardiogram and HR extraction algorithms. Therefore, our study targets comparing four types of HR extraction algorithms on the FOS mattress, namely MODWT, CEEMDAN, cepstrum and clustering. The goal is to select, based on their accuracy and computational speed, the most suitable one for online or offline application purposes. Results of applying these four chosen algorithms on the FOS mattress show that the cepstrum is the most accurate algorithm with a mean absolute error (MAE) of $4.62 \pm 1.68$ BPM. However, the cepstrum is more appropriate for offline monitoring with a runtime of $662.9 \mathrm{~ms}$ for a 10second signal segment. The results also show that the Maximal Overlap Direct Wavelet Transform (MODWT) is more efficient with a runtime of $\mathbf{4 . 1}$ milliseconds for online purposes, but with a slightly bigger MAE $(6.87 \pm 1.94$ BPM). Both methods proved to be as efficient on the new mattress technology as past intelligent mattresses.
\end{abstract}

Index Terms-Biomedical monitoring, Biomedical transducers, Error analysis, Performance analysis, Estimation, Signal processing algorithms, Heart rate

\section{INTRODUCTION}

Monitoring of a patient's vital signs is crucial. Vital signs are paramount to establish accurate diagnoses over medical problems a person may have. It has been proven, by Donaldson et al.[1], that diagnoses from short time monitoring are a source of medical errors. These errors happen when long-term information is missing. As a consequence, it is recommended to have continuous monitoring of the vital signs to ensure an optimal diagnosis of a patient's state [2]. A new generation of sensor-based mattress is able to unobtrusively monitor vital signs such as the heart rate (HR). These intelligent mattresses (e.g., based on strain gauge, piezoelectric, fiber bragg grating,

This research was supported by the Natural Sciences and Engineering Research Council - Undergraduate Student Research Awards (BRPC-5132432017) and the Canada Research Chair on Biomedical Data Mining (950231214).

978-1-5386-8173-2/18/\$31.00 (C)2018 IEEE

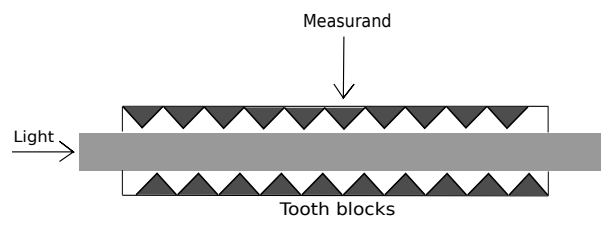

Fig. 1: Microbend FOS principle. The light passing through the microbend FOS is modulated by the deformations in the optical fiber due to the displacement of the micro-benders.

etc.) have shown promising results in extracting the HR. The strain gauge or hydraulic sensor [3] was mostly used to monitor respiratory and cardiac activity as well as body movements. The piezoelectric sensor [4][5] implemented in a mattress can also monitor the HR. These intelligent mattresse solutions often either lack sensitivity or are too expensive. Recently, an interesting microbend fiber optic sensor (FOS) has been developed. This microbend FOS provides a new way of acquiring the mechanical activity of the human body. It is achieved through the intensity attenuation of the light passing through an optic fiber in response to a mechanical stimulus on the fiber [6]. Figure 1 shows the principle of the microbend FOS.

The FOS sensor is sensitive to small movements and has the ability to retrieve the ballistocardiogram (BCG) signal. This signal is present in the range of indiscernible motions coming from the human body. The BCG measures the ballistic forces generated by the heart, that is, the mechanical response of the body when the heart ejects the blood into the vascular tree. Analog to the electrocardiogram (ECG) and its well-known QRS complex, the BCG possesses the IJK complex, as seen in figure 2. It is, therefore, possible to extract information like the interval between two heartbeats to characterize the heart rate. However, this signal is corrupted because of the so-called motion artifacts. Filtrating the motion artifacts that occur when a person makes even the slightest movement requires advanced signal processing techniques.

In this work, we experiment the performances of this new mattress by applying four types of algorithms to retrieve the 


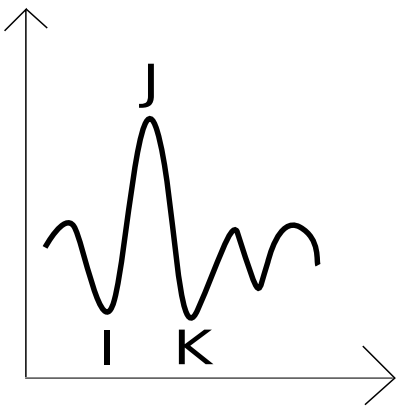

Fig. 2: Shape of a BCG signal and the IJK complex.

HR from the motions sensed on the FOS mattress, namely complete ensemble empirical mode decomposition with adaptative noise (CEEMDAN) [7], clustering [8], cepstrum [3], and the Maximal overlap direct wavelet transform (MODWT). The MODWT is the only one already applied on a microbend FOS mattress in a previous work [9]. Our aim is to recommend the methods that are more adapted to offline or online signal processing applications in the case of heart rate extraction.

Following, we review in section 2 the four main families of algorithms used in extracting the HR. In section 3, we describe how we implemented these algorithms from these four main families. In section 4, we present the results of applying these algorithms on the FOS mattress with 6 healthy subjects. In section 5, we assess the performances of the new mattress according to these advanced signal processing techniques. Subsequently, it is possible to propose recommendations for future use of the mattress depending on the required computational speed and accuracy of the application.

\section{RELATED WORKS}

The biggest sources of noise in extracting the BCG are the motion artifacts. These artifacts interfere with the measurements when the subject does even the smallest motion. To extract the BCG in all these sources of noise, numerous digital signal processing tools have been used. In this study, we investigated the following four main families of signal processing tools: 1) Wavelet transform (WT), 2) Empirical mode decomposition (EMD), 3) Clustering and 4) Cepstrum. Our study is limited to these algorithms because they are representative of their respective families and the most used in the literature.

\section{A. Wavelet transform}

WT is often used for signal denoising as well as for feature extraction. Its strength relies on its ability to split the signal into multiple frequency components. As a result, we gain a spectro-temporal representation of this signal. Jin et al. [10] detected the heart rate with a peak searching algorithm using Donoho and Johnstone's [11] wavelet shrinking method with a Symlet-8 wavelet basis. Sadek et al. [9] implemented the MODWT to extract the BCG signal from a microbend FOS.

\section{B. Empirical Mode Decomposition}

The EMD is a modified method derived from the Hilbert Transform called the Hilbert-Huang transform [12]. This method decomposes a given signal in finite and smaller "intrinsic mode functions"(IMF). It then allows seeing instantaneous frequencies as a function of time. Pinheiro et al. [13] also decomposed the BCG time series into few components and found the $\mathrm{BCG}$ in motionless recordings to recover part of the heartbeat information. Following the ensemble EMD (EEMD) procedure, Song et al. [14] extracted the BCG for cardiovascular classification. Sadek et al. [7] used an enhanced version of EEMD, CEEMDAN, to extract the BCG from an FBG sensor. At the ninth decomposition component of the CEEMDAN, they retrieved the heart rate with an error as little as $6.81 \pm 1.15 \mathrm{BPM}$.

\section{Clustering}

Machine learning (ML), with its recent popularity, has also been applied to extract vital signs from an unobtrusive sensor. Cluster analysis, a branch of ML, examines unlabeled samples, by either constructing a hierarchical structure or forming a set of groups. Clustering is generally used to train a prototype model of the heartbeat. Brüser et al. [8] extracted the heart rate with a $k$-means clustering algorithm. Paalasmaa and Ranta [15] also used a clustering algorithm but with an ideal signal which they generated.

\section{Cepstrum}

The cepstrum is a processing technique often used for speech processing in the domain of the quefrencies [16]. The cepstrum is the inverse Fourier transform of the logarithmic spectrum of a signal. With this representation, we can uncover the fundamental periods of a signal, that is, the time occurrence of certain signals including HR. The cepstrum has been applied with good results to estimate the heart rate. Brüser et al. [3] recorded the mean of the spectrum on different sensors with a sliding window. They then converted it to the cepstrum domain. Zhu et al. [17] used the same approach by extracting the cepstrum from a sliding window algorithm. They then filtered the cepstrum to make easier finding the heart rate peak.

In the next section, we explain the methods we used to extract the HR from an FOS mattress.

\section{Methodology}

We present in this section our experimental setup with the FOS mattress and the approach we used to assess its performances. We also examine the custom peak-searching algorithm used to extract the HR from the BCG, as well as the metric used to compare the algorithms we implemented.

The FOS mattress was fixed on the back of a regular office chair, as shown in figure 3. The system used for collecting data includes a small FOS mattress and a module to gather optical data coming from the mattress. The raw data is sampled at $50 \mathrm{~Hz}$ by the module. As a ground truth, this study uses the clinically validated Hexoskin intelligent textile (see figure 4). The Hexoskin is embedded with cardiac sensors giving 


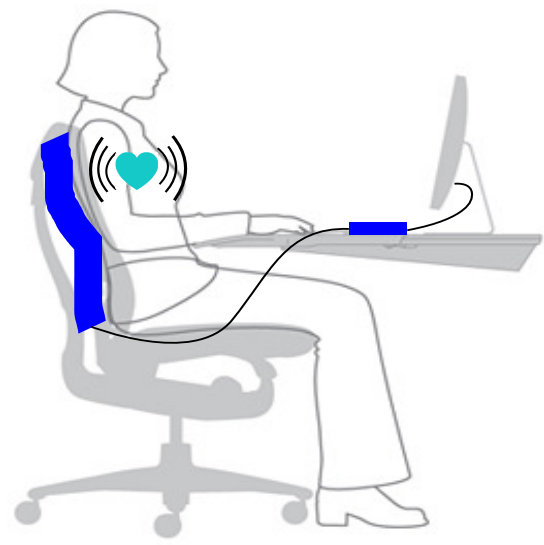

Fig. 3: Experimental setup

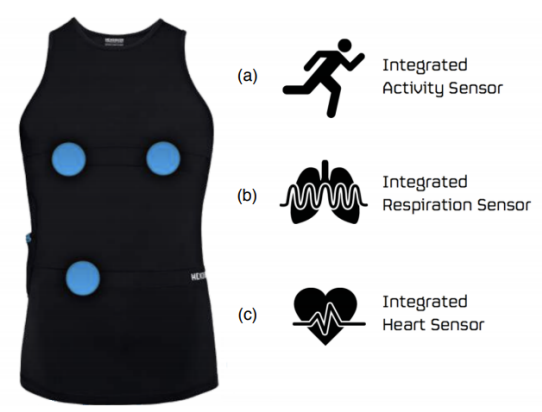

Fig. 4: The Hexoskin smart textile

an accurate HR of its wearer [18][19]. During the whole experiment, the subject was wearing the Hexoskin and was asked to sit still on the chair for a duration of 5 minutes. During this time, a Raspberry Pi 3B records the data streamed on the serial port of the FOS's module. Figure 5 shows an example of BCG signal and ECG reference signal. The whole data is subsequently processed in MATLAB R2017a with the exception of the MODWT algorithm being implemented using python 3.5. In total, 30 minutes of recordings are used to compare methods across themselves.

\section{A. Preprocessing}

All raw signals were preprocessed with a bidirectional Butterworth bandpass filter ranging from 0.5 to $10 \mathrm{~Hz}$. The exception being CEEMDAN with a high pass frequency of 1.0 $\mathrm{Hz}$ because it was more sensitive to low-frequency respiratory artifacts.

\section{B. Peak searching algorithm}

To extract the HR from the BCG, we apply a custom peak-searching algorithm inspired from Mack et al. [20] on the MODWT, CEEMDAN, clustering and cepstrum methods, using a 10-seconds sliding window. A peak extraction algorithm is necessary since the HR extraction methods being compared do not extract the heart rate, but only the BCG. We used a peak-searching algorithm that sets higher and lower boundaries to the HR [17][20]. The peak detection threshold is automatically adjusted if the quantity of detected peaks is outside boundaries. This algorithm is shown at algorithm 1

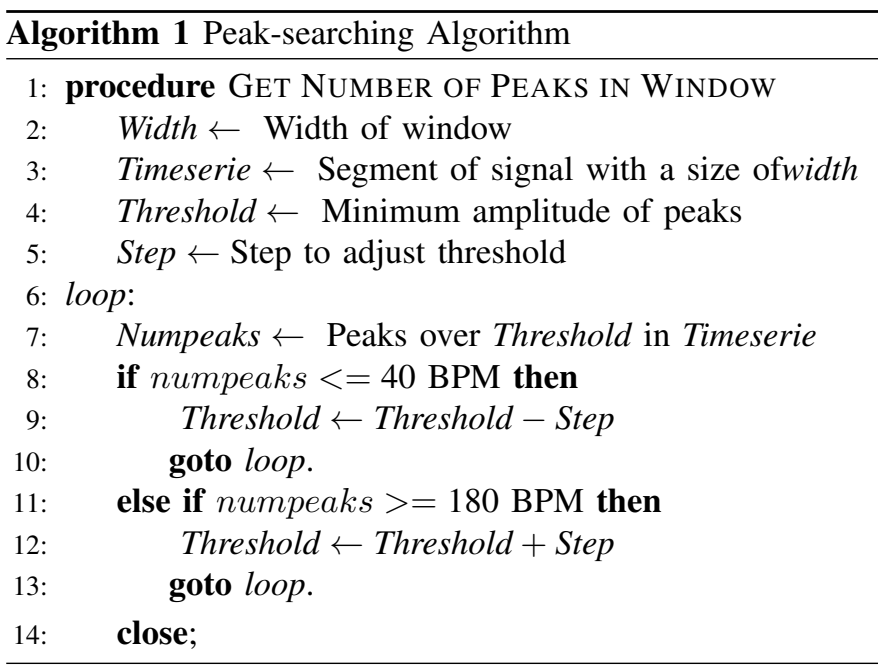

\section{HR extraction methods}

1) MODWT: MODWT is one of the methods that yielded good results in the past. We exploited MATLAB to implement MODWT. The approach we use is similar to that of Sadek et al. [9]. The 4th level smooth coefficients of the wavelet decomposition are used to extract the BCG. We then apply the peak searching algorithm described earlier.

2) CEEMDAN: The literature also revealed frequent methods making use of the EMD. The CEEMDAN is an optimized method frequently used for EMD. The CEEMDAN, in particular, shows excellent results in extracting the HR from still subjects [9]. We use the CEEMDAN algorithm provided by Torres et al. [21] and we set the parameters according to a noise standard deviation of 0.2 , a number of realizations of 100 and a maximum number of iterations of 30[7]. Finally, we apply the same custom peak searching algorithm.

3) Clustering: As for the third method, we apply the ML method proposed by Brüser et al. [8] for the FOS mattress. We take features of the signal and then use Principal Component Analysis (PCA) to reduce their dimensionality. Subsequently, we use clustering to create groups with the two most important principal components as the axes. The resulting feature prototype, which should describe best a heartbeat, is thus returned. Our implementation uses only the cross correlation between the prototype to find group of features akin to a heartbeat. That is, a local maxima is most related to a heartbeat when the correlation is big. Subsequently, we extract the peaks of the signal to characterize the HR.

4) Cepstrum: The cepstrum method as seen increased interest in extracting vital signs. Using the cepstrum of the filtered data, we implement the method of Zhu et al.[17] on the FOS mattress. That is, we transform a sliding 10 -seconds window for each segment of the signal to the cepstral domain. For each 

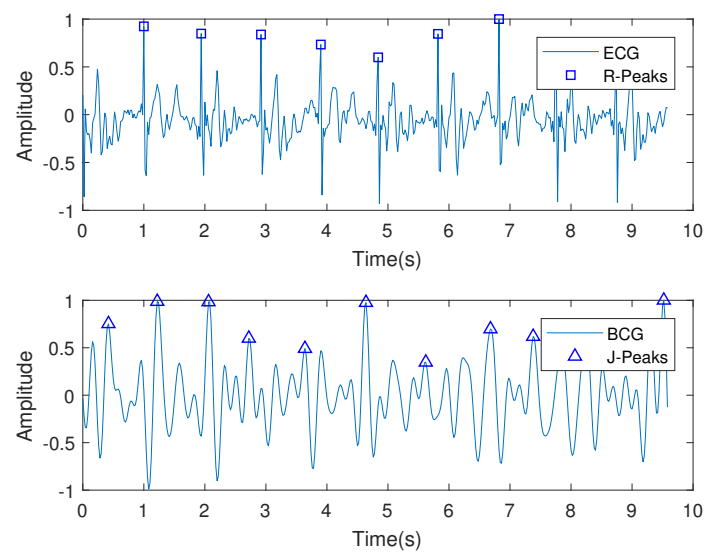

Fig. 5: An example of ECG reference signal with R-Peaks, compared to BCG signal with J-Peaks

transformed window, we look for the highest peak between a period of 40 and 180 BPM [17].

\section{Experimental setup}

This study was reviewed and approved by the TELUQ, ETS and CHUM institutional review board (IRB). We recruited 6 subjects, three men and three women, each between 20 and 35 years old from the CHUM research center by a general invitation. Subjects are all in good health and have no prior medical conditions that may impact the quality of their vital signs.

\section{E. Validation}

The first measure used to assess the quality of each method is the Mean Absolute Error (MAE). This quantity is used in statistics to measure how close a prediction is to the actual outcome.

$$
M A E=\frac{1}{n} \sum\left|y_{i}-\widehat{y_{i}}\right|
$$

where

$$
y_{i}=\text { Ground truth value and } \widehat{y_{i}}=\text { Estimated value }
$$

The second measure, the Computational Speed (CS), is used to quantify the time cost of the operations realized by the system while running one of the algorithms. We record the time before and after running the algorithm to estimate the execution time.

In the next section, we examine the results of the four algorithms we implemented and explain their performances.

\section{RESULTS AND DISCUSSION}

For each method, we extract the MAE from the HR measurements in order to quantify the deviation from the ground truth. We also measure CS for a 10-second segment of the signal on the 6 subjects to compare time efficiency across methods (table I).

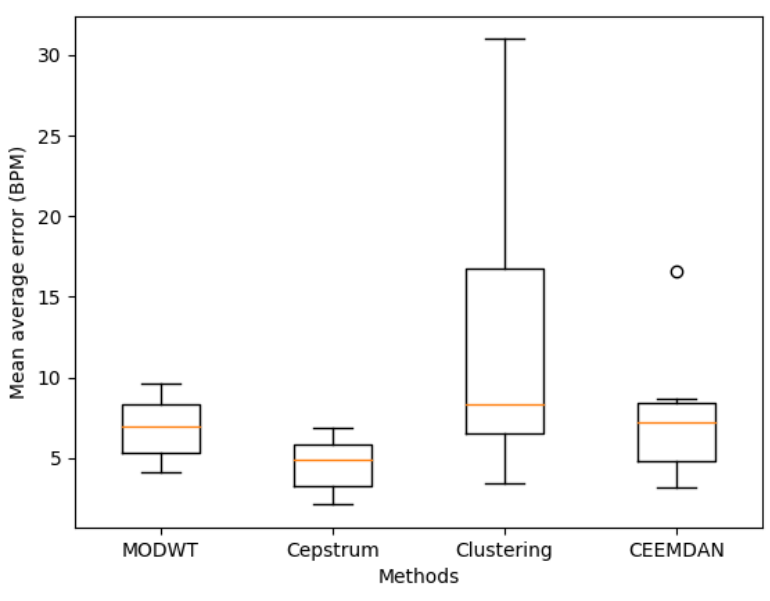

Fig. 6: MAE on all subjects for all methods

\begin{tabular}{cccc}
\hline Subject & MAE(Average) & MAE(Std) & CS(ms)(Average) \\
\hline \hline MODWT & 6.87 & 1.94 & 4.1 \\
\hline Cepstrum & 4.62 & 1.68 & 662.9 \\
\hline Clustering & 12.76 & 9.53 & 99.6 \\
\hline CEEMDAN & 7.85 & 4.34 & 2236.7 \\
\hline
\end{tabular}

TABLE I: Average performance of the algorithms on all subjects. MAE is in beats per minute (BPM)

For each algorithm, the MAE is computed in beats per minute (BPM) and the computational speed (CS) in milliseconds (ms). For MODWT, the error $(6.87 \pm 1.94 \mathrm{BPM})$ is one of the smallest. These results confirm the findings of Sadek et al. [9], showing that MODWT has good efficiency in extracting clear heartbeat peaks from the BCG. A simple peak searching algorithm applied to the output of the wavelet transform is enough to obtain satisfying results. Woever, it sometimes detects more or fewer peaks than there really is. This is due to the simplistic search of peaks and could use more peak selection heuristics. Nonetheless, it offers the best CS from all with an excellent $4.1 \mathrm{~ms}$. This time performance for MODWT is due to the signal going through an equivalent bank of high pass and low pass filter when being processed, thus making it faster than other algorithms.

CEEDMAN has higher MAE results (7.85 $44.34 \mathrm{BPM})$ than MODWT and has a much higher CS with a runtime of 2236.7 ms because it needs to perform several iterations to get the result. These performances are lower but in accordance with the results of Sadek et al. [7]. This difference could be due to the information about the HR being in more than one intrinsic mode function.

As for clustering, we observe that it has the worst MAE among all methods, (12.76 $\pm 9.53 \mathrm{BPM})$. Results are worse than what Brüser et al. found [8] because we used only the cross-correlation in the processing step. It is, therefore, important to implement fusion of HR markers. These markers include cross-correlation results, euclidean distance and the heart valve component as mentioned by Brüser et al. [8]. 
Even with marker fusion, accuracy is still low because of the difficulty in selecting the right cluster to represent the heart beat. Currently, the values for clustering at table I show that it is less preferable due to the poor accuracy obtained during the experiment.

The cepstrum offers the best precision $(4.62 \pm 1.68 \mathrm{BPM})$ of all. These results are even better than those from Zhu et al. [17]. On the other hand, it is slower $(662.9 \mathrm{~ms})$ than most methods because it must go through both direct and inverse Fourier transforms. Still, this speed is not critical since it would only apply an additional time shift in the estimated heart rate in online applications. Using the same algorithm with a GPU could increase the CS by a good margin. For all methods, the peak searching algorithm should not only set lower and higher boundaries but also filter intermediate peaks. This filtering is to avoid counting a peak that comes too soon after a sequence of equally-spaced peaks.

Finally, MODWT is more convenient with a runtime of $4.1 \mathrm{~ms}$ for online applications. Yet, clustering offers the best trade-off in terms of robustness and computational cost. It is, therefore, a better choice for offline analysis of the heart rate variability.

\section{CONClusion}

Continuous and unobtrusive monitoring of vital signs is imperative for establishing rigorous diagnoses. In this work, we measured the performances of a new generation of fiber optic sensor (FOS) mattress technology. We used four known methods of heart rate extraction from the ballistocardiogram (BCG) signal: 1) MODWT, 2) CEEMDAN, 3) Clustering and 4) Cepstrum. Our goal was to assess the performances of the new mattress according to these advanced signal processing techniques, in order to propose better recommendations for future use of the mattress.

According to our experiments, we can classify each of these methods according to 1) the mean absolute error (MAE) of the heart rate estimate with a valid ground truth and 2) the computational time required to run said algorithms. Results show that maximal overlap direct wavelet transform (MODWT) and cepstrum show the best results among the four tested algorithms. However, cepstrum has more computational time and less MAE than MODWT and the others. It would thus be more adapted to offline purposes whereas MODWT would be a better choice for online applications. These results establish good reasons for using the novel FOS technology for continuous vital signs monitoring.

Considering these findings, the FOS mattress could be adapted to the clinical context for better diagnoses. We are working on a new study that involves a higher number of subjects in a clinical context in order to help detect pathologies such as heart failure. By acquiring a high quantity of data, we will be able to use more robust machine learning algorithms.

\section{ACKNOWLEDGMENT}

This research was supported in partby the Natural Science and Engineering Research Council (BRPC-513243-2017) and the Canada Research Chair on Biomedical Data Mining (950231214). The authors would like to thank the anonymous reviewers for their valuable comments and suggestions to improve the quality of the paper

\section{REFERENCES}

[1] M. S. Donaldson, J. M. Corrigan, L. T. Kohn, and others, To err is human: building a safer health system, vol. 6. National Academies Press, 2000.

[2] J. Liu, Y. Wang, Y. Chen, J. Yang, X. Chen, and J. Cheng, "Tracking vital signs during sleep leveraging off-the-shelf WiFi," in Proceedings of the 16th ACM International Symposium on Mobile Ad Hoc Networking and Computing, MobiHoc '15, (New York, NY, USA), pp. 267-276, ACM, 2015

[3] C. Bruser, J. M. Kortelainen, S. Winter, M. Tenhunen, J. Pärkkä, and S. Leonhardt, "Improvement of Force-Sensor-Based Heart Rate Estimation Using Multichannel Data Fusion," IEEE Journal of Biomedical and Health Informatics, vol. 19, pp. 227-235, Jan. 2015.

[4] T. Koivistoinen, S. Junnila, A. Varri, and T. Koobi, "A new method for measuring the ballistocardiogram using EMFi sensors in a normal chair," in The 26th Annual International Conference of the IEEE Engineering in Medicine and Biology Society, vol. 1, pp. 2026-2029, Sept. 2004.

[5] Y. Katz, R. Karasik, and Z. Shinar, "Contact-free piezo electric sensor used for real-time analysis of inter beat interval series," in 2016 Computing in Cardiology Conference (CinC), pp. 769-772, Sept. 2016.

[6] E. Udd, "An overview of fiber-optic sensors," review of scientific instruments, vol. 66, no. 8, pp. 4015-4030, 1995.

[7] I. Sadek, J. Biswas, V. F. S. Fook, and M. Mokhtari, "Automatic heart rate detection from FBG sensors using sensor fusion and enhanced empirical mode decomposition," in 2015 IEEE International Symposium on Signal Processing and Information Technology (ISSPIT), pp. 349353, Dec. 2015.

[8] C. Bruser, K. Stadlthanner, S. d. Waele, and S. Leonhardt, "Adaptive Beat-to-Beat Heart Rate Estimation in Ballistocardiograms," IEEE Transactions on Information Technology in Biomedicine, vol. 15, pp. 778-786, Sept. 2011.

[9] I. Sadek, J. Biswas, B. Abdulrazak, Z. Haihong, and M. Mokhtari, "Continuous and unconstrained vital signs monitoring with ballistocardiogram sensors in headrest position," in 2017 IEEE EMBS International Conference on Biomedical Health Informatics (BHI), pp. 289-292, Feb. 2017.

[10] J. Jin, X. Wang, S. Li, and Y. Wu, "A Novel Heart Rate Detection Algorithm in Ballistocardiogram Based on Wavelet Transform," in 2009 Second International Workshop on Knowledge Discovery and Data Mining, pp. 76-79, Jan. 2009.

[11] D. L. Donoho and I. M. Johnstone, "Adapting to Unknown Smoothness via Wavelet Shrinkage," Journal of the American Statistical Association, vol. 90, p. 1200, Dec. 1995.

[12] N. E. Huang, Z. Shen, S. R. Long, M. C. Wu, H. H. Shih, Q. Zheng, N.C. Yen, C. C. Tung, and H. H. Liu, "The empirical mode decomposition and the Hilbert spectrum for nonlinear and non-stationary time series analysis," Proceedings of the Royal Society of London A: Mathematical, Physical and Engineering Sciences, vol. 454, pp. 903-995, Mar. 1998.

[13] E. C. Pinheiro, O. A. Postolache, and P. S. Girão, "Online heart rate estimation in unstable ballistocardiographic records," in 2010 Annual International Conference of the IEEE Engineering in Medicine and Biology, pp. 939-942, Aug. 2010.

[14] Y. Song, H. Ni, X. Zhou, W. Zhao, and T. Wang, "Extracting Features for Cardiovascular Disease Classification Based on Ballistocardiography," in 2015 IEEE 12th Intl Conf on Ubiquitous Intelligence and Computing and 2015 IEEE 12th Intl Conf on Autonomic and Trusted Computing and 2015 IEEE 15th Intl Conf on Scalable Computing and Communications and Its Associated Workshops (UIC-ATC-ScalCom), pp. 1230-1235, Aug. 2015.

[15] J. Paalasmaa and M. Ranta, "Detecting heartbeats in the ballistocardiogram with clustering," in Proceedings of the ICML/UAI/COLT 2008 Workshop on Machine Learning for Health-Care Applications, Helsinki, Finland, vol. 9, 2008.

[16] A. V. Oppenheim and R. W. Schafer, "From frequency to quefrency: A history of the cepstrum," IEEE signal processing Magazine, vol. 21, no. 5, pp. 95-106, 2004. 
[17] Y. Zhu, V. F. S. Fook, E. H. Jianzhong, J. Maniyeri, C. Guan, H. Zhang, E. P. Jiliang, and J. Biswas, "Heart rate estimation from FBG sensors using cepstrum analysis and sensor fusion," in 2014 36th Annual International Conference of the IEEE Engineering in Medicine and Biology Society, pp. 5365-5368, Aug. 2014.

[18] C. A. Sayed, L. Vinches, and S. Hallé, "Validation of a Wearable Biometric System's Ability to Monitor Heart Rate in Two Different Climate Conditions under Variable Physical Activities," E-Health Telecommunication Systems and Networks, vol. 06, p. 19, June 2017.

[19] N. H. Cherif, N. Mezghani, N. Gaudreault, Y. Ouakrim, I. Mouzoune, and P. Boulay, "Physiological data validation of the hexoskin smart textile," in 11th International Conference on Biomedical Electronics and Devices, pp. 150-156, Jan. 2018.

[20] D. C. Mack, J. T. Patrie, P. M. Suratt, R. A. Felder, and M. Alwan, "Development and Preliminary Validation of Heart Rate and Breathing Rate Detection Using a Passive, Ballistocardiography-Based Sleep Monitoring System," IEEE Transactions on Information Technology in Biomedicine, vol. 13, pp. 111-120, Jan. 2009.

[21] M. E. Torres, M. A. Colominas, G. Schlotthauer, and P. Flandrin, "A complete ensemble empirical mode decomposition with adaptive noise," pp. 4144-4147, IEEE, May 2011. 